\title{
SPIN STABILIZED SATELLITE'S ATTITUDE PROPAGATION WITH QUATERNIONS
}

\author{
M. C. Zanardi \\ UNESP $^{-}$São Paulo State University, \\ Guaratinguetá , SP, CEP 12516-410 -BRAZIL,55-12- 3123-2845 \\ cecília@feg.unesp.br
}

\section{Orlando}

INPE- Brazilian National Institute for Space Research, São José dos Campos , SP, CEP 12201-970 - BRAZIL, 55- 12- 3208-6374 valcir@ccs.inpe.br

\section{R. S. P. Bento}

UNESP $^{-}$São Paulo State University, Guaratinguetá , SP, CEP 12516-410 -BRAZIL,55-12- 3123-2845

\author{
M. F. Silva \\ UNESP $^{-}$São Paulo State University, \\ Guaratinguetá , SP, CEP 12516-410 -BRAZIL,55-12- 3123-2845
}

\begin{abstract}
This paper presents a study of a modeling scheme for the spin stabilized satellites attitude, entirely developed in terms of quaternion parametrization. The analysis includes numerical propagation of the rotational motion equation, considering the influence of the following torques: aerodynamic, gravity gradient, residual magnetic, eddy currents and the one due to the Lorentz force. Applications are developed considering the Brazilian Spin Stabilized Satellites SCD1 and SCD2, which are quite appropriated for verification and comparison of the theory with the real data generated and processed by the INPE's Satellite Control Center (SCC). The results show that for SCD1 and SCD2 the influence of the eddy current torque is bigger than the others ones, not only due to the orbit altitude, but also to other specific satellites characteristics. The influence of the torque due to Lorentz force is smaller than the others ones because of the dimension and the electrical charges of the SCD1 and SCD2. In all performed tests the errors remained within the dispersion range specified for the attitude determination system of INPE's SCC. The results show the feasibility of using the quaternion attitude parametrization for modeling the satellite dynamics of spin stabilized satellites.
\end{abstract}

Keywords: attitude quaternion, spin velocity, external torques, numerical simulation, pointing deviation.

\section{Introduction}

The objective of this paper is to analyze the attitude of spin stabilized satellites, entirely developed in terms of quaternion parametrization. The analysis includes numerical propagation of the rotational motion equation, considering the influence of the following torques: aerodynamic, gravity gradient, residual magnetic, eddy currents and the one due to the Lorentz force.

The gravity gradient torque is generated by the difference of the Earth gravity force direction and the intensity actuating on each satellite mass element. This torque is inversely proportional to the cube of the satellite geocentric distance. The aerodynamic torque is created by the interactions of rarefied air particles with the satellite surface and it has the predominant orbit perturbation effect in LEO orbit satellites. In this paper TD-88 model is used to describe the atmospheric density. The residual magnetic torque results from the interaction between the spacecraft's residual magnetic moment and the Earth magnetic field and its main effect is to produce a spin axis orientation drift. On the other hand, the main effect of the eddy current torque is to produce a reduction in the satellite spin rate with time. The torque due to Lorentz force is associated with a rigid spacecraft equipped with an electrostatically charged protective shield, having an intrinsic magnetic moment. The main element of this shield is an electrostatically charged screen surrounding the protected volume of the spacecraft. This torque depends on the Earth's magnetic field, the form of the satellite shell, the satellite spin rate, the angular velocity of the diurnal rotation of the geomagnetic field altogether with the Earth and the electrical 
charge of the satellite. In this paper the dipole model is assumed for the Earth's magnetic field and the satellite is supposed to be in an elliptical orbit.

A mathematical model is presented for each considered torque in terms of quaternion attitude parametrization.

The numerical propagation of the equations of rotational motion shows the evolution of the components of the angular velocity vector and the four components of the attitude quaternion. The influences of the Earth oblateness in the orbital elements are taken into account. Applications are developed considering the Brazilian spin stabilized satellites SCD1 and SCD2, which are quite appropriated for verification and comparison of the theory with the real data generated and processed by the INPE's Satellite Control Center (INPE's SCC). A spherical coordinate system fixed in the satellite is used to locate the satellite spin axis in relation to the terrestrial equatorial system. The spin axis direction is specified by its right ascension and the declination angles. The time evolution of the spin axis right ascension and declination angles is gotten from the numerical results of the quaternion attitude propagation.

An initial approach is presented, in which the propagated attitude is daily updated with the help of real satellite data, supplied by INPE's SCC. A second approach is also presented, where daily updates of the attitude data has not been performed in the propagation process.

The results of this analysis can be useful for the Brazilian mission satellite.

\section{External torques model}

\subsection{Gravity gradient torque}

The gravity gradient torque (Vilhena de Moraes, 1994; Zanardi and Real, 2003) for a spacecraft can be modeled by:

$$
\vec{M}=3 \frac{\mu}{r^{\prime 3}}\left[a_{21} a_{31}\left(I_{z}-I_{y}\right) \hat{e}_{x}+a_{11} a_{31}\left(I_{x}-I_{z}\right) \hat{e}_{y}+a_{11} a_{21}\left(I_{y}-I_{x x}\right) \hat{e}_{z}\right]
$$

where $\mu\left(3.986 \times 10^{14} \mathrm{~m}^{3} / \mathrm{s}^{2}\right)$ is the Earth gravitational parameter, $r^{\prime}$ is the satellite geocentric distance, $\mathrm{a}_{11}, \mathrm{a}_{21}$ and $\mathrm{a}_{31}$ are the direction cosines which relate the orbital system and the satellite fixed system (the latter being associated with the principal moments of inertia axes of the satellite), $I_{x}, I_{y}, I_{z}$ are the Principal Moments of Inertia of the satellite and $\hat{e}_{x}, \hat{e}_{y}, \hat{e}_{z}$ units vectors of the satellite fixed system. The elements $a_{11}, a_{21}$ and $a_{31}$ depend on the orbital elements (orbit inclination, true anomaly, longitude of the ascending node and argument of the perigee) and the attitude quaternion (Zanardi and Real, 2003; Zanardi et al., 2010). In this study the z-axis corresponds to the long axis of the cylinder. Equation (1) shows that this torque decreases with the cube of the altitude and depends on the shape, dimension and mass distribution of the satellite. If the satellite has a uniform mass distribution and the principal moments of inertia are equal, this torque vanishes. The gravity gradient torque magnitude has short oscillations due to the satellite rotational motion (Zanardi and Real, 2003) when the influence of the rotational motion is included in the direction cosines $\mathrm{a}_{11}, \mathrm{a}_{21}$ and $\mathrm{a}_{31}$.

\subsection{Aerodynamic torque}

When the satellites move in the tenuous layers of the upper atmosphere, the interactions of the molecular stream with a satellite's surface produces a torque about the center of mass. For spacecrafts below approximately 400 $\mathrm{km}$, the aerodynamic torque is the dominant environmental disturbance torque (Wertz, 1978; Zanardi and Real, 2003). The end of a spacecraft mission often occurs when the aerodynamic torques becomes so great that the spacecraft reenters the Earth's atmosphere and the attitude control systems ceases to become effective and the spacecraft tumbles.

In this paper we will adopt, to represent the aerodynamic torque, the following model (Lafontaine and Mamem, 1984):

$$
\overrightarrow{N_{A}}=\overrightarrow{m_{e}} \times \vec{D}
$$

Where $\overrightarrow{\boldsymbol{m}_{\boldsymbol{e}}}$ is the position vector between the center of pressure and the center of mass, the $\overrightarrow{\boldsymbol{D}}$ is the drag force (in this paper the influence of the lift force in the aerodynamic torque is negligenciable). In the satellite fixed system it is given by: 


$$
\vec{D}=D_{x} \hat{e}_{x}+D_{y} \hat{e}_{y}+D_{z} \hat{e}_{z}
$$

With:

$$
\begin{aligned}
& D_{x}=-D\left[a_{11} \cos \left(\gamma_{S}\right)+a_{12} \operatorname{sen}\left(\gamma_{S}\right)\right] \\
& D_{y}=-D\left[a_{21} \cos \left(\gamma_{S}\right)+a_{22} \operatorname{sen}\left(\gamma_{S}\right)\right] \\
& D_{z}=-D\left[a_{31} \cos \left(\gamma_{S}\right)+a_{32} \operatorname{sen}\left(\gamma_{S}\right)\right] \\
& D=\frac{1}{2} \rho v^{2} S C_{D}
\end{aligned}
$$

where $\rho$ is the local density, $v$ represents the magnitude of the satellite's velocity relative to the atmosphere, $S$ is a reference section area of the satellite, $C_{D}$ is the Drag Coefficient, $\gamma_{S}$ is the angle between the position vector and the orbital velocity vector and $a_{\mathrm{ij}}, \mathrm{i}=1,2,3, \mathrm{j}=1,2$, are the direction cosines which relate the orbital system and the satellite fixed system and depend on the orbital elements and the attitude quaternion (Zanardi et al., 2010). An analysis concerning the uncertainties and usual values of some of these parameters can be found in Lafontaine and Mamem (1984).

Then, by substituting Eq. 3 in Eq. 2, the aerodynamic torque in the satellite fixed system is given by:

$$
\vec{N}_{A}=\left[D_{z} m e_{y}-D_{y} m e_{z}\right] \hat{e}_{x}+\left[D_{x} m e_{z}-D_{z} m e_{x}\right] \hat{e}_{y}+\left[D_{x} m e_{z}-D_{z} m e_{x}\right] \hat{e}_{z}
$$

In order to estimate the influence of the aerodynamic torque magnitude in the rotational motion in this paper some simplifications are done and the thermosphere model TD-88 is used for the atmospheric density (Sehnal and Pospísilová, 1988). The velocity $v$ is assumed to be equal to the orbital velocity and the drag coefficient is fixed. The thermosphere model TD-88 is defined for the height range of $150-750 \mathrm{~km}$. According to Sehnal and Pospísilová (1988), the atmospheric density on a surface of constant altitude can be described by the expression:

$$
\rho=f_{x} f_{0} k_{0} \sum_{n=1}^{7} h_{n} g_{n}
$$

where:

$$
h_{n}=K_{n, 0}+\sum_{j=1}^{3} K_{n, j} \exp \left[\frac{\left(120-\left(r^{\prime}-r_{t}\right)\right.}{29 j}\right]
$$

Here, $\mathrm{r}_{\mathrm{t}}$ is Earth's Equatorial radius; $K_{n, j}$ are numerical constants; $f_{x}$ and $f_{0}$ depend on the solar flux and $k_{0}$ depends on the geomagnetic index. The terms $g_{n}$ (describing mean density, individual dependence on the mean solar flux, North-South asymmetry, annual, semi-annual, diurnal and semi-diurnal variations) are functions of the day count of the year, of the local time, of the latitude, and the numerical constants which are summarized in tables (Sehnal and Pospísilová, 1988).

\subsection{Magnetic torques}

Magnetic disturbance torques result from the interaction between the spacecraft's residual magnetic field and the Earth's magnetic field. In this paper it is assumed that the spacecraft is manufactured from material such that the primary sources of magnetic torques are the spacecraft magnetic moments and eddy currents with other negligible sources. The spacecraft's magnetic moment is usually the dominant source between the disturbances torques (Vilhena de Moraes, 1994). If $\vec{m}$ is the magnetic moment of the spacecraft and $\vec{B}$ is the geocentric 
magnetic flux density, the residual magnetic torques is given by Garcia et al. (2009), Zanardi et al. (2005) and Zanardi and Real (2003):

$$
\vec{N}_{r}=\vec{m} \times \vec{B}
$$

The torque induced by eddy currents is caused by the spacecraft spinning motion. It is known (Garcia et al. (2009) and Zanardi et al. (2005)) that the eddy currents produce a torque which causes the precession in the spin axis and causes an exponential decay of the spin rate. If $\vec{W}$ is the spacecraft's angular velocity vector and $\mathrm{p}$ is a constant coefficient which depends on the spacecraft geometry and conductivity, this torque is given by:

$$
\vec{N}_{i}=p \vec{B} \times(\vec{B} \times \vec{W})
$$

Here the magnetic torque is developed only for a spin-stabilized satellite. In this case, the spacecraft's angular velocity vector and the satellite magnetic moment are along the z-axis and the residual magnetic torque and the induced eddy currents can the expressed in the satellite fixed system by (Garcia et al., 2009):

$$
\begin{aligned}
& \vec{N}_{r}=-m B_{y} \hat{e}_{x}+m B_{x} \hat{e}_{y} \\
& \vec{N}_{i}=p W\left(B_{x} B_{z} \hat{e}_{x}+B_{y} B_{z} \hat{e}_{y}-\left(B_{x}{ }^{2}+B_{y}{ }^{2}\right) \hat{e}_{z}\right)
\end{aligned}
$$

where $B_{x}, B_{y}, B_{z}$ are the components of the geomagnetic field in the satellite fixed system (Zanardi et al., 2009). These components are obtained in terms of the geocentric inertial components of the geomagnetic field (Thomas and Cappelari, 1964) and the attitude quaternion of the satellite (Zanardi et al., 2009). In order to describe the geomagnetic field, the dipole vector model (Thomas and Cappelari ,1964; Zanardi et al., 2005) is used.

\subsection{Torque due Lorentz force}

In this paper we will adopt the following model to represent the torque due to the Lorentz force (Abdel-Aziz, 2007):

$$
\overrightarrow{M_{L}}=\vec{W} \times \overrightarrow{\beta_{S}}+\overrightarrow{w_{g}} \times \overrightarrow{\beta_{S}}
$$

where $\vec{W}$ is the spin velocity vector of the satellite; $\overrightarrow{w_{g}}$ is the vector of the spin velocity of the diurnal rotational of the geomagnetic field together with the Earth, with the direction given by the unit vector $\hat{\beta}$ and

$$
\overrightarrow{\beta_{S}}=S \hat{\beta}, \quad S=\left(\begin{array}{ccc}
4 D d^{2} & 0 & 0 \\
0 & 4 D d^{2} & 0 \\
0 & 0 & 4 D h^{2}
\end{array}\right), \quad \quad \quad \quad \text { and } \quad \frac{Q B}{4 r^{\prime 3}}
$$

where $d$ and $h$ are the diameter and height of the cylindrical charged manta around the satellite, $B$ is the magnitude of the geomagnetic field, $r^{\prime}$ is the satellite geocentric distance and $Q$ is the satellite's electrical charge. The geomagnetic field is described by the dipole vector model (Thomas and Cappelari ,1964; Zanardi et al., 2005).

After the development of Eq. 15, the components of the torque due to the Lorentz force in the satellite fixed satellite is (Bento, 2010):

$$
\vec{M}_{L}=M_{L x} \widehat{e}_{x}+M_{L y} \widehat{e}_{y}+M_{L z} \widehat{e}_{z}
$$

with

$$
\begin{aligned}
& M_{L x}=D h^{2} \beta_{3 P}\left(q+w_{g} \beta_{2 P}\right)-D d^{2} \beta_{2 P}\left(r+w_{g} \beta_{3 P}\right), \\
& M_{L y}=D d^{2} \beta_{1 P}\left(r+w_{g} \beta_{3 P}\right)-D h^{2} \beta_{3 P}\left(p+w_{g} \beta_{1 P}\right),
\end{aligned}
$$




$$
M_{L z}=D d^{2}\left(p \beta_{2 P}-q \beta_{1 P}\right),
$$

where $\mathrm{p}, \mathrm{q}, \mathrm{r}$ are the components of the satellite spin velocity $\vec{W}$ in the satellite fixed system, $\beta_{1 \mathrm{p}}, \beta_{2 \mathrm{p}}, \beta_{3 \mathrm{p}}$, depend on the components of $\hat{\beta}$ in the equatorial system and the attitude quaternion.

\section{Equations of rotational motion in terms of the quaternion}

The dynamic equations of the satellite's rotational motion are described by the Euler equations and the kinematic equations for the attitude quaternion. The Euler equations give the rate of variation of the components of the satellite's spin velocity and depend on the components of the external torques in the satellite fixed system (Pisacane and Moore, 1994; Vilhena de Moraes, 1994; Zanardi et al., 2009):

$$
\begin{aligned}
& \dot{p}=q r\left(I_{y}-I_{z}\right) / I_{X}+N_{X} / I_{X}, \\
& \dot{q}=p r\left(I_{z}-I_{X}\right) / I_{y}+N_{y} / I_{y}, \\
& \dot{r}=p q\left(I_{X}-I_{y}\right) / I_{z}+N_{z} / I_{z} .
\end{aligned}
$$

In these equations $I_{x}, I_{y}, I_{z}$ are the Principal Moments of Inertia of the satellite, p,q,r and $N_{x}, N_{y}, N_{z}$ are the components of the spin velocity and the external torques in the satellite fixed system, respectively.

In this paper the kinematic equations are described in terms of the attitude quaternion $q$. The quaternion $q$ is a vector $4 \times 1$ given by:

$$
q=\left[\begin{array}{llll}
q_{1} & q_{2} & q_{3} & q_{4}
\end{array}\right]^{t}=\left[\begin{array}{ll}
\vec{q} & q_{4}
\end{array}\right]^{t},
$$

where $t$ represents the transposed of the matrix. It is usual to call the vector $\vec{q}$ as the vector component of the quaternion and $q_{4}$ the scalar component of the quaternion. They can be expressed as a function of the rotation angle $(\phi)$ and of the axis of rotation $\vec{n}$ as shown below:

$$
\vec{q}=\left[\begin{array}{lll}
q_{1} & q_{2} & q_{3}
\end{array}\right]^{t}=\sin (\phi / 2) \vec{n} \text { and } q_{4}=\cos (\phi / 2) .
$$

It is ease to prove that the module of the quaternion is 1 , since $\vec{n}$ is a unitary vector in the direction of the spin velocity vector. The matrix of attitude in terms of the quaternion is presented in Pisacane and Moore (1994). The kinematic equations that describe the rate of variation of the components of the quaternion of attitude, due to rotation of the satellite, are given by Pisacane and Moore (1994) and Zanardi et al. (2009):

$$
\begin{array}{ll}
\dot{q}_{1}=\frac{1}{2}\left[p q_{4}-q q_{3}+r q_{2}\right], & \dot{q}_{2}=\frac{1}{2}\left[q q_{4}-r q_{1}+p q_{3}\right], \\
\dot{q}_{3}=\frac{1}{2}\left[r q_{4}-p q_{2}+q q_{1}\right], & \dot{q}_{4}=-\frac{1}{2}\left[p q_{1}+q q_{2}+r q_{3}\right] .
\end{array}
$$

As it can be observed, the kinematic equations in terms of the attitude quaternion are linked and depend on the components $(p, q, r)$ of the spin velocity, given by the Eq. 21, making it difficult to get an analytical solution. Then, in this paper, a numerical propagation of the Eq. 21 and Eq. 24 is applied, using the Runge Kutta method and the language FORTRAN.

\section{Applications for spin stabilized satellite}

Spin stabilized satellites has the spin axis along the biggest principal moment of inertia's axis. A spherical coordinates system fixed in the satellite is used to locate the spin axis of the satellite with respect to the equatorial system. The direction of the spin axis $\hat{k}$ is specified by its right ascension $(\alpha)$ and the declination $(\delta)$ which are represented in Fig. 1.This spherical coordinate can be obtained using the attitude quaternion $q$ and the components of the spin velocity $W$. If the unit vectors $(\hat{I}, \hat{J}, \widehat{K})$ are associated with the equatorial system and the units vectors $\hat{e}_{x}, \hat{e}_{y}, \hat{e}_{z}$ of the satellite fixed system then the spin velocity vector can be expressed by: 


$$
\overrightarrow{W_{I}}=P \hat{I}+Q \hat{J}+R \widehat{K} \quad \text { and } \quad p \widehat{e_{x}}+q \widehat{e_{y}}+r \widehat{e_{z}}
$$

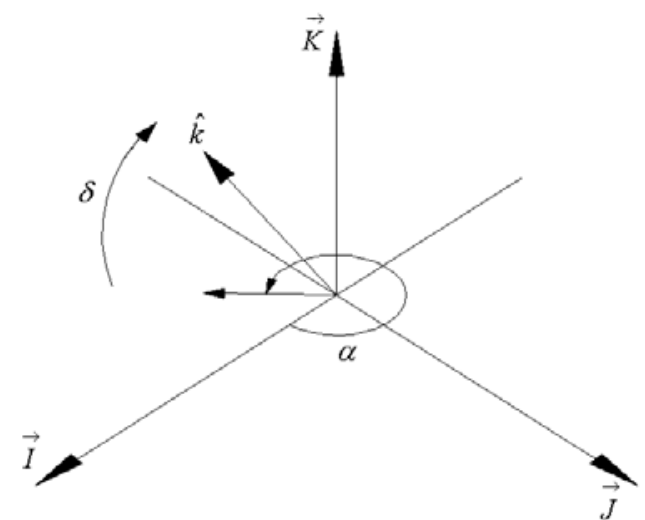

Figure 1. Equatorial System ( $\hat{\mathrm{I}}, \hat{\mathrm{J}}, \hat{\mathrm{K}})$, spin axis orientation $(\hat{\mathrm{k}})$, right ascension $(\alpha)$ and declination $(\delta)$ of the spin axis

If the components of the attitude quaternion $\left(q_{1}, q_{2}, q_{3}, q_{4}\right)$ and components of the satellite spin velocity $(p, q, r)$ are known, the vectors $\vec{W}$ and $\overrightarrow{W_{I}}$ are related by (Zanardi et al., 2009):

$$
\begin{aligned}
& P=\left(q_{1}^{2}-q_{2}^{2}-q_{3}^{2}+q_{4}^{2}\right) p+2 q\left(q_{2} q_{1}-q_{4} q_{3}\right)+2 r\left(q_{3} q_{1}+q_{4} q_{2}\right) \\
& Q=2 p\left(q_{1} q_{2}+q_{4} q_{3}\right)+\left(-q_{1}^{2}+q_{2}^{2}-q_{3}^{2}+q_{4}^{2}\right) q+2 r\left(q_{3} q_{2}-q_{4} q_{1}\right) \\
& R=2 p\left(q_{3} q_{1}-q_{4} q_{2}\right)+2 q\left(q_{3} q_{2}+q_{4} q_{1}\right)+\left(-q_{1}^{2}-q_{2}^{2}+q_{3}^{2}+q_{4}^{2}\right) r
\end{aligned}
$$

and the magnitude of the spin velocity is given by:

$$
W=\left(p^{2}+q^{2}+r^{2}\right)^{\frac{1}{2}}
$$

According to Fig. 1, the components of spin velocity P. Q, R can be obtained by:

$$
P=W \cos \delta \cos \alpha, \quad Q=W \cos \delta \sin \alpha, \quad R=W \sin \delta .
$$

Then the right ascension ( $\alpha$ ) and declination $(\delta)$ of the spin velocity can be computed by:

$$
\sin \delta=\frac{R}{W}, \quad \cos \propto=\frac{P}{W \cos \delta}, \quad \sin \alpha=\frac{Q}{W \cos \delta}
$$

with $0 \leq \delta \leq 90^{\circ}$ and $0 \leq \alpha \leq 360^{\circ}$.

In this paper the right ascension and declination of the spin velocity will be computed using the numerical results obtained for the components of the spin velocity and attitude quaternion by the numerical simulation of the Eqs. 21 and Eq. 24.

\section{Pointing deviation}

For the tests it is important to observe the deviation between the actual attitude data supplied by INPE and the computed attitude for each satellite. Here this deviation is called pointing deviation and it is given by the angle $\theta$ between the actual spin axis $\hat{k}$ and the computed spin axis $\widehat{k_{c}}$. It can be computed by (Garcia et al., 2009):

$$
\cos \theta=\hat{k} \cdot \widehat{k_{c}}
$$

where $(\cdot)$ indicates the scalar product between actual spin axis $\hat{k}$ and computed spin axis $\widehat{k_{c}}$.

The unit vectors $\hat{k}$ and $\widehat{k_{c}}$ can be obtained using the right ascension and declination of the spin axis as: 


$$
\begin{aligned}
\widehat{k} & =\cos \alpha \cos \delta \hat{I}+\sin \alpha \cos \delta \hat{J}+\sin \delta \widehat{K}, \\
\widehat{k_{c}} & =\cos \alpha_{c} \cos \delta_{C} \hat{I}+\sin \alpha_{c} \cos \delta_{C} \hat{J}+\sin \delta_{C} \widehat{K},
\end{aligned}
$$

with $\alpha$ and $\delta$ supplied by INPE's SCC and $\alpha_{c}$ and $\delta_{c}$ computed by the presented theory.

\section{Numerical applications}

The theory developed here has been applied to the spin stabilized Brazilian Satellite (SCD1 and SCD2) for verification and comparison of the theory against the data generated by the INPE's SCC. The $8^{\text {th }}$ Runge Kutta method is used to determine the numerical solution for Eq. 21 and Eq. 24.

The numerical solutions give the components of the attitude quaternion and of the spin velocity, which are used to compute the spin velocity, right ascension and declination of the spin axis by using Eq. 27 and Eq. 29. Then, these computed values are compared with real data supplied by INPE's SCC in order to check the precision of the presented theory. It is also important to observe the deviation between the actual spin axis and the computed spin axis, that is, the pointing deviation computed by Eq. 30 .

Two approaches are presented. In the first one the propagated attitude is daily updated with the help of actual satellite data, supplied by INPE's SCC. In the second approach the daily updates of the attitude data has not been performed in the propagation process. In both approaches the orbital elements are updated, taking into account the main influences of the Earth oblateness.

Initial conditions for the attitude were taken from INPE supplied data (Garcia et al., 2009; Vilhena de Moraes, 1994; Zanardi and Real, 2003).

\subsection{First approach: daily updated data}

Simulations for the SCD1 were made for 17 days. The results for the deviation between the computed values and actual values for right ascension, declination and spin velocity and the pointing deviation are shown in Tab. 1 for the case that considers all torques actuating together. In Fig. 2, Fig. 3, Fig. 4 and Fig. 5 are shown the results for the deviation between the computed values and the actual values of the right ascension, declination and spin velocity and for the pointing deviation when it is considered each torque individually and all torques actuating together. In Tab. 2 are shown the mean values for each simulation.

Table 1. Deviation between computed values and actual values when all torques actuating together for SCD1, with the daily updated data

\begin{tabular}{|c|c|c|c|c|}
\hline Day & $\begin{array}{c}\text { Deviation in } \\
\text { Right Ascension } \\
\text { (degrees) }\end{array}$ & $\begin{array}{c}\text { Deviation in } \\
\text { Declination } \\
\text { (degrees) }\end{array}$ & $\begin{array}{c}\text { Deviation in } \\
\text { Spin Velocity } \\
\text { (rpm) }\end{array}$ & $\begin{array}{c}\text { Pointing } \\
\text { Deviation } \\
\text { (degrees) }\end{array}$ \\
\hline $\mathbf{1 7 / 8 / 1 9 9 3}$ & 0.000 & 0.000 & 0.000 & 0.000 \\
\hline $\mathbf{1 8 / 8 / 1 9 9 3}$ & 0.870 & -0.347 & -0.021 & 0.373 \\
\hline $\mathbf{1 9 / 8 / 1 9 9 3}$ & 0.662 & -0.350 & -0.016 & 0.366 \\
\hline $\mathbf{2 0 / 8 / 1 9 9 3}$ & 0.385 & -0.358 & -0.026 & 0.364 \\
\hline $\mathbf{2 1 / 8 / 1 9 9 3}$ & 0.154 & -0.361 & -0.033 & 0.362 \\
\hline $\mathbf{2 2 / 8 / 1 9 9 3}$ & -0.079 & -0.338 & -0.034 & 0.338 \\
\hline $\mathbf{2 3 / 8 / 1 9 9 3}$ & -0.232 & -0.328 & -0.061 & 0.33 \\
\hline $\mathbf{2 4 / 8 / 1 9 9 3}$ & 0.654 & -0.172 & -0.057 & 0.21 \\
\hline $\mathbf{2 5 / 8 / 1 9 9 3}$ & -0.657 & -0.308 & 0.010 & 0.332 \\
\hline $\mathbf{2 6 / 8 / 1 9 9 3}$ & -0.759 & -0.281 & 0.010 & 0.317 \\
\hline $\mathbf{2 7 / 8 / 1 9 9 3}$ & -1.596 & -0.031 & -0.078 & 0.314 \\
\hline $\mathbf{2 8 / 8 / 1 9 9 3}$ & -0.192 & -0.452 & 0.057 & 0.454 \\
\hline $\mathbf{2 9 / 8 / 1 9 9 3}$ & -1.041 & -0.200 & -0.020 & 0.292 \\
\hline $\mathbf{3 0 / 8 / 1 9 9 3}$ & -1.068 & -0.184 & -0.027 & 0.288 \\
\hline $\mathbf{3 1 / 8 / 1 9 9 3}$ & -1.110 & -0.147 & -0.033 & 0.276 \\
\hline $\mathbf{1 / 9} / \mathbf{1 9 9 3}$ & -1.163 & -0.119 & -0.022 & 0.274 \\
\hline $\mathbf{2 / 9 / 1 9 9 3}$ & -1.178 & -0.090 & -0.025 & 0.268 \\
\hline
\end{tabular}




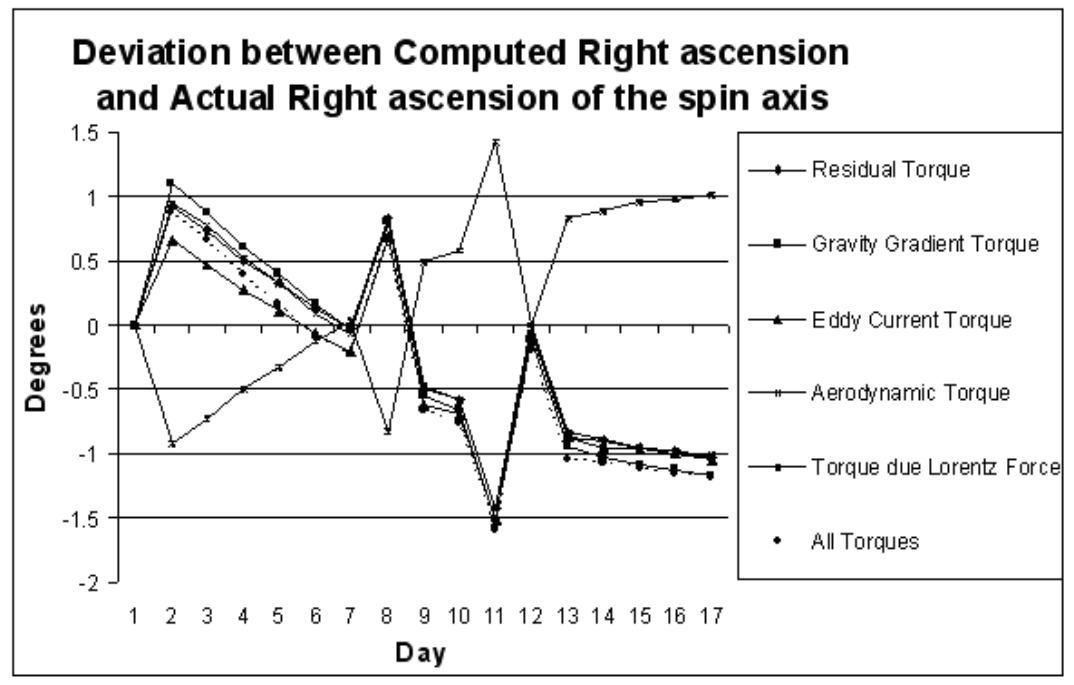

Figure 2. Temporal variation for the deviation between the computed and actual right ascension of the spin axis for SCD1 and with the daily updated data

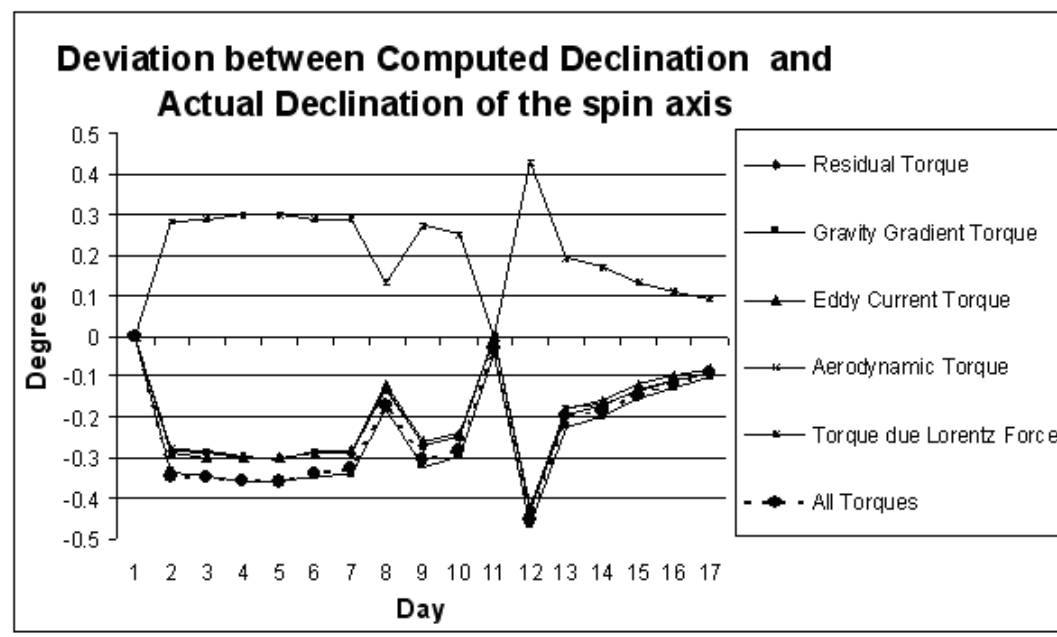

Figure 3. Temporal variation for the deviation between the computed and actual declination of the spin axis for SCD1 and with the daily updated data

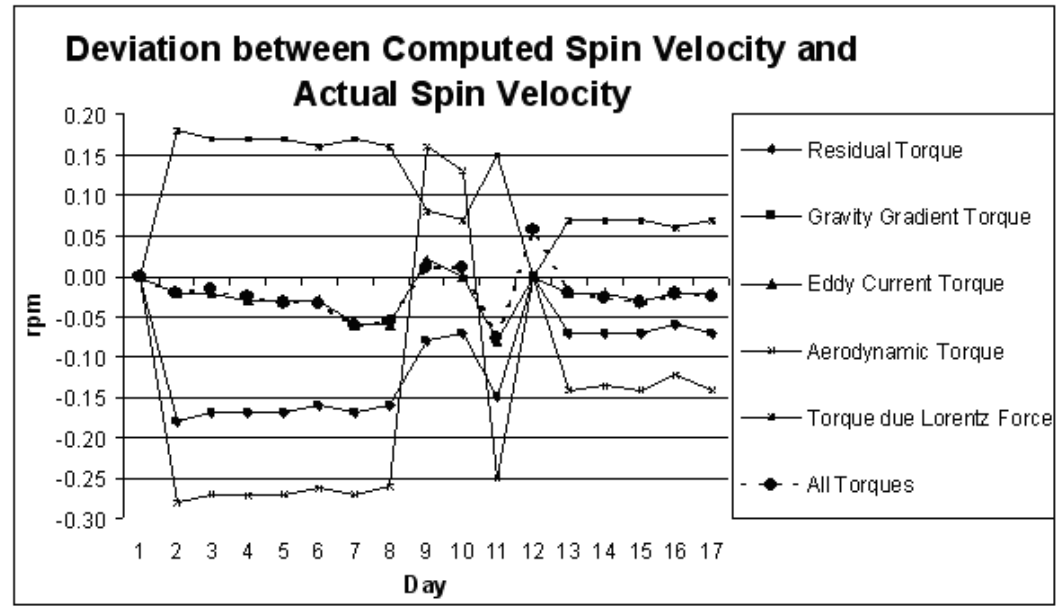

Figure 4. Temporal variation for the deviation between the computed and actual spin velocity for SCD1 and with the daily updated data 


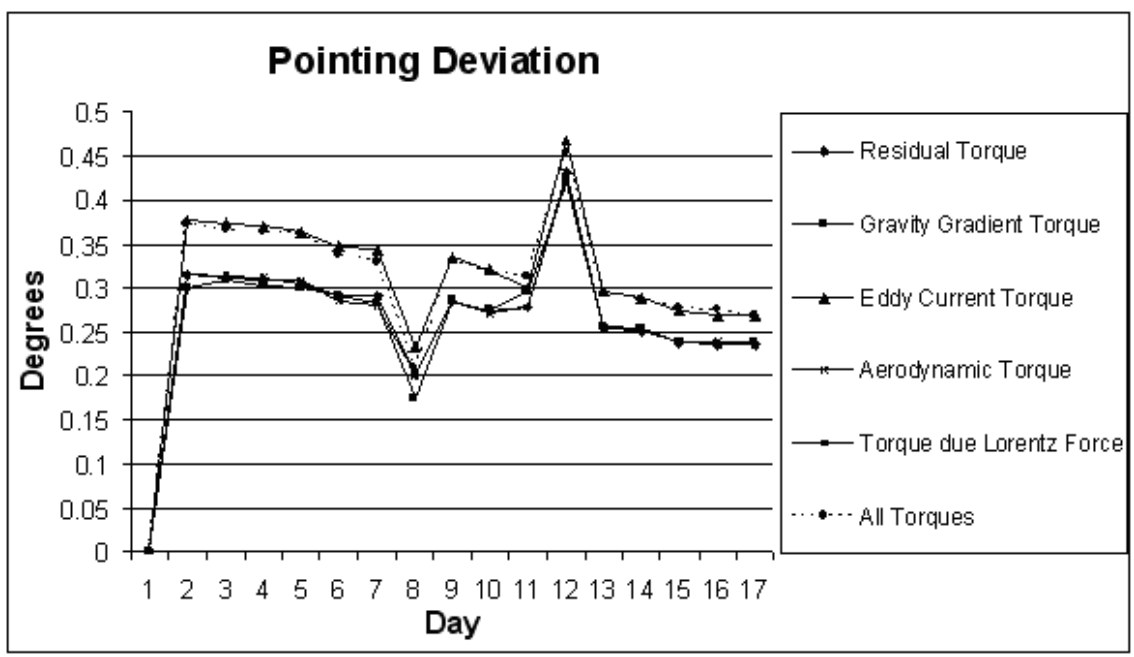

Figure 5. Temporal variation for the pointing deviation for SCD1 and with the daily updated data

For the test period of 17 days, the results show that the mean deviation error in right ascension, declination, spin velocity and pointing deviation are within the dispersion range of the attitude determination system performance of INPE's control center, which is $0.5^{\circ}$ for the angles and $0.5 \mathrm{rpm}$ for the spin velocity.

The residual torque acts in the opposite direction of the torque due to the Lorentz force. The biggest influence in spin velocity and in the declination of the spin axis is given by the eddy currents torque, with the mean deviation equals to $-0.025 \mathrm{rpm}$ and $-0.202^{\circ}$, respectively.

Table 2. Mean values for SCD1 simulations with the daily updated data

\begin{tabular}{|l|c|c|c|c|c|c|}
\hline \multicolumn{1}{|c|}{$\begin{array}{c}\text { Included } \\
\text { Torques }\end{array}$} & Residual & $\begin{array}{c}\text { Gravity } \\
\text { Gradient }\end{array}$ & $\begin{array}{c}\text { Eddy } \\
\text { current }\end{array}$ & $\begin{array}{c}\text { Due } \\
\text { Lorentz } \\
\text { force }\end{array}$ & $\begin{array}{c}\text { All } \\
\text { torques }\end{array}$ \\
\hline $\begin{array}{l}\text { Right Ascension } \\
\text { Deviation (degrees) }\end{array}$ & -0.221 & -0.251 & -0.341 & -0.225 & 0.221 & -0.374 \\
\hline $\begin{array}{l}\text { Declination Deviation } \\
\text { (degrees) }\end{array}$ & -0.207 & -0.247 & -0.202 & -0.205 & 0.207 & -0.239 \\
\hline $\begin{array}{l}\text { Spin Velocity } \\
\text { Deviation (rpm) }\end{array}$ & -0.107 & -0.107 & -0.025 & -0.148 & 0.107 & -0.022 \\
\hline $\begin{array}{l}\text { Pointing Deviation } \\
\text { (degrees) }\end{array}$ & 0.265 & 0.262 & 0.308 & 0.264 & 0.265 & 0.303 \\
\hline
\end{tabular}

Simulations for the SDC2 were made for 16 days. The results for the deviation between the computed values and actual values for right ascension, declination and spin velocity and the pointing deviation are shown in Tab.3 for the case that considers all torques actuating together. In Fig. 6, Fig. 7, Fig. 8 and Fig. 9 are shown the results for the deviation between the computed values and actual values of the right ascension, declination and spin velocity and for the pointing deviation when it is considered each torque individually and all torques actuating together. The discontinuities in these figures occur due to the attitude control corrections applied by SCC. In Tab. 4 are shown the mean and standard deviation for each simulation. It is important to note that, when the attitude control actuates, the computed values are assumed to be equal to the real data because the control system is not included in the proposed theory.

For the test period of 16 days, the mean deviation error are also within the dispersion range of the attitude determination system of INPE's control center. The biggest influence in spin velocity is given by eddy currents torque, with the mean deviation error equal to $-0.009 \mathrm{rpm}$. The biggest influence in the right ascension and declination of the spin axis is due to the gravity gradient torque, with the mean deviation error equal to $0.001^{\circ}$ and $0.002^{\circ}$, respectively. 
Table 3. Deviation between computed values and actual values when all torques actuating together for SCD2, with daily updated data

\begin{tabular}{|c|c|c|c|c|}
\hline Day & $\begin{array}{c}\text { Deviation in } \\
\text { Right } \\
\text { Ascension } \\
\text { (degrees) }\end{array}$ & $\begin{array}{c}\text { Deviation in } \\
\text { Declination } \\
\text { (degrees) }\end{array}$ & $\begin{array}{c}\text { Deviation in } \\
\text { Spin Velocity } \\
\text { (rpm) }\end{array}$ & $\begin{array}{c}\text { Pointing } \\
\text { Deviation } \\
\text { (degrees) }\end{array}$ \\
\hline $\mathbf{1 2 / 2 / 2 0 0 2}$ & 0.000 & 0.000 & 0.000 & 0.000 \\
\hline $\mathbf{1 3 / 2 / 2 0 0 2}$ & 0.023 & -0.018 & -0.012 & 0.021 \\
\hline $\mathbf{1 4 / 2 / 2 0 0 2}$ & 0.023 & 0.004 & 0.000 & 0.011 \\
\hline $\mathbf{1 5 / 2 / 2 0 0 2}$ & 0.029 & 0.012 & -0.007 & 0.018 \\
\hline $\mathbf{1 6 / 2 / 2 0 0 2}$ & 0.031 & 0.018 & 0.004 & 0.023 \\
\hline $\mathbf{1 7 / 2 / 2 0 0 2}$ & 0.028 & 0.024 & -0.004 & 0.027 \\
\hline $\mathbf{1 8 / 2 / 2 0 0 2}$ & 0.040 & 0.036 & -0.004 & 0.04 \\
\hline $\mathbf{1 9 / 2 / 2 0 0 2}$ & 0.061 & 0.060 & -0.002 & 0.066 \\
\hline $\mathbf{2 0 / 2 / 2 0 0 2}$ & 0.087 & 0.086 & -0.002 & 0.094 \\
\hline $\mathbf{2 1 / 2 / 2 0 0 2}$ & 0.119 & 0.088 & -0.001 & 0.103 \\
\hline $\mathbf{2 2 / 2 / 2 0 0 2}$ & 0.126 & 0.095 & -0.002 & 0.111 \\
\hline $\mathbf{2 3 / 2 / 2 0 0 2}$ & 0.108 & 0.095 & -0.013 & 0.107 \\
\hline $\mathbf{2 4 / 2 / 2 0 0 2}$ & 0.000 & 0.000 & 0.000 & 0.000 \\
\hline $\mathbf{2 5 / 2 / 2 0 0 2}$ & 0.059 & -0.087 & 0.051 & 0.092 \\
\hline $\mathbf{2 6 / 2 / 2 0 0 2}$ & 0.038 & -0.084 & -0.093 & 0.086 \\
\hline $\mathbf{2 7 / 2 / 2 0 0 2}$ & 0.033 & -0.096 & -0.028 & 0.097 \\
\hline
\end{tabular}

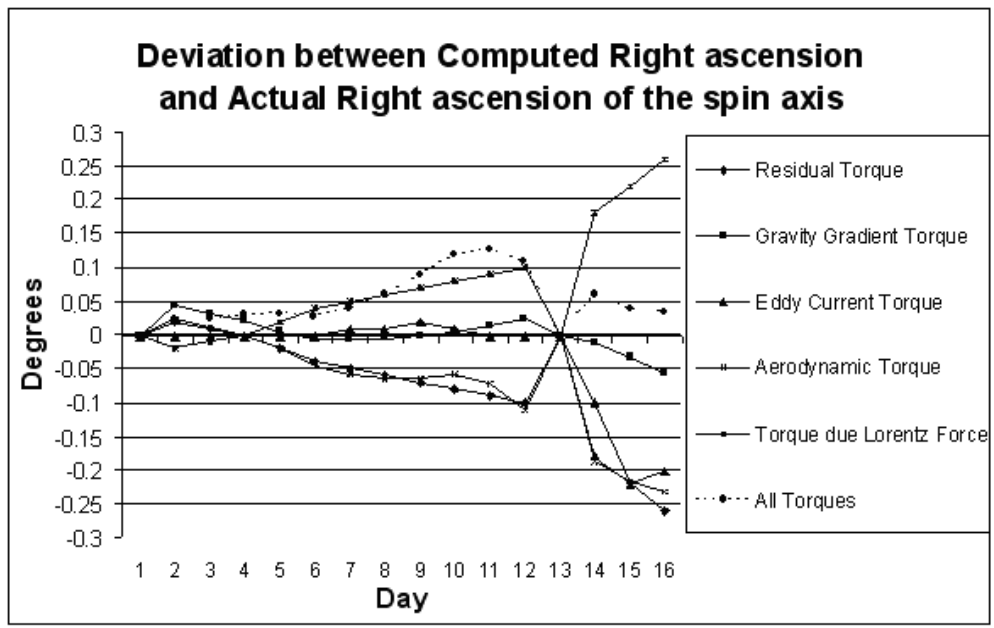

Figure 6. Temporal variation for the deviation between the computed and actual right ascension of the spin axis for SCD2 and with the daily updated data

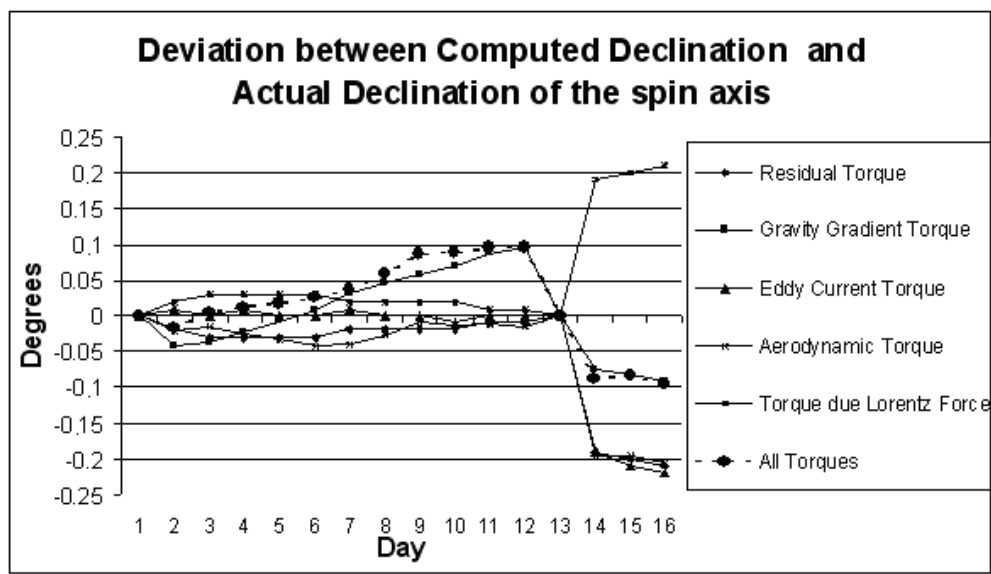

Figure 7. Temporal variation for the deviation between the computed and actual declination of the spin axis for SCD2 and with the daily updated data 


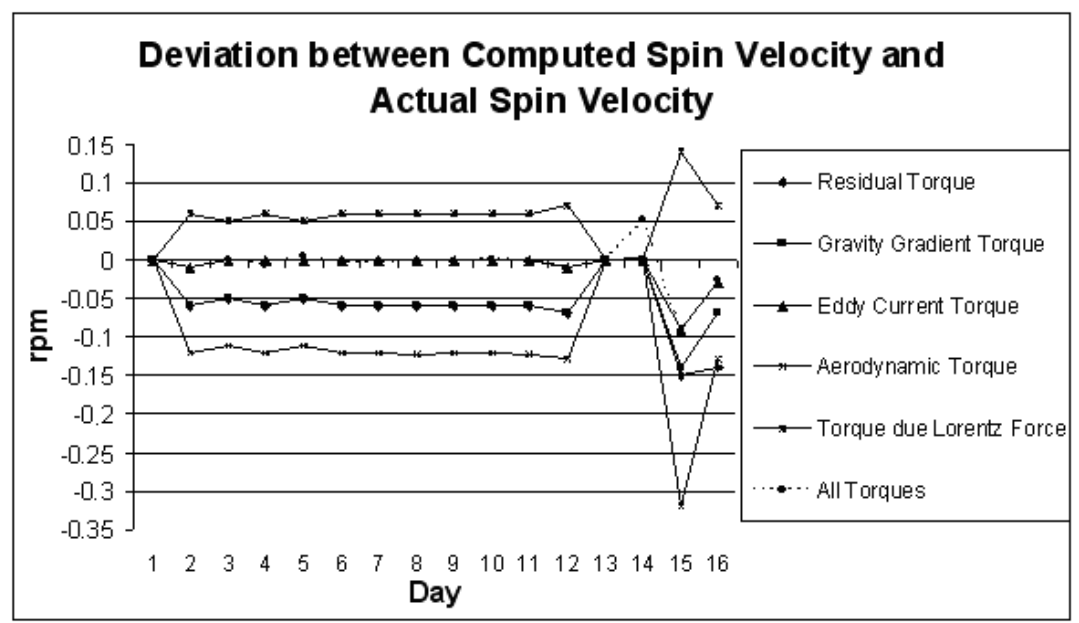

Figure 8. Temporal variation for the deviation between the computed and actual spin velocity for SCD2 and with the daily updated data

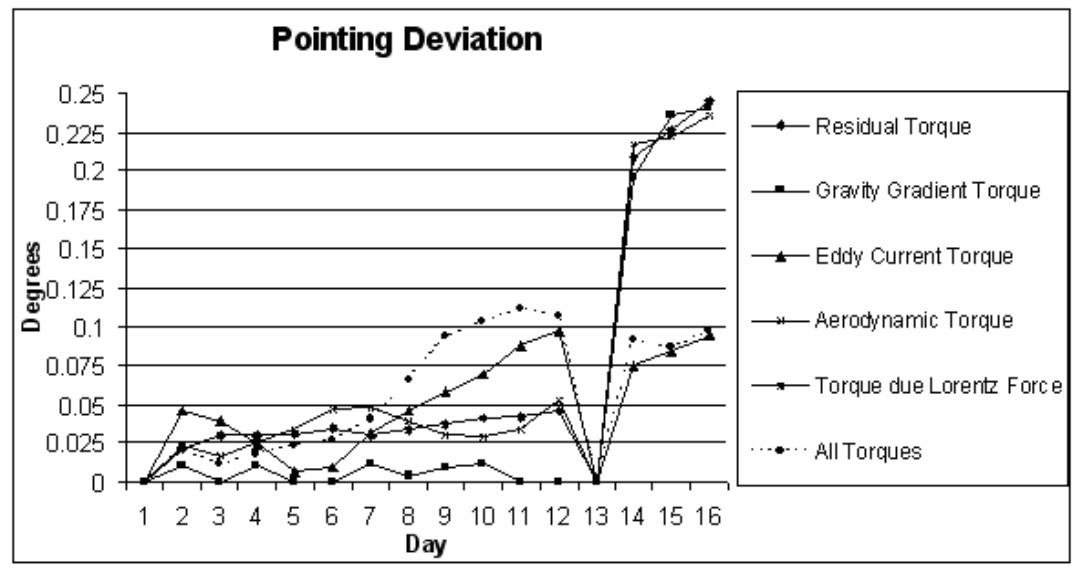

Figure 9. Temporal variation for the pointing deviation for SCD2 and with the daily updated data

Table 4. Mean values for SCD2 simulations with the daily updated data

\begin{tabular}{|c|c|c|c|c|c|c|}
\hline $\begin{array}{c}\text { Included } \\
\text { Torques }\end{array}$ & Residual & $\begin{array}{c}\text { Gravity } \\
\text { Gradient }\end{array}$ & $\begin{array}{c}\text { Eddy } \\
\text { current }\end{array}$ & $\begin{array}{c}\text { Due } \\
\text { Lorentz } \\
\text { force }\end{array}$ & $\begin{array}{c}\text { All } \\
\text { torques }\end{array}$ \\
\hline $\begin{array}{c}\text { Right Ascension } \\
\text { Deviation (degrees) }\end{array}$ & -0.071 & 0,001 & $-0,029$ & -0.068 & 0.071 & 0.050 \\
\hline $\begin{array}{c}\text { Declination } \\
\text { Deviation (degrees) }\end{array}$ & $-0,053$ & 0,002 & $-0,037$ & -0.053 & 0.056 & 0.015 \\
\hline $\begin{array}{c}\text { Spin Velocity } \\
\text { Deviation (rpm) }\end{array}$ & $-0,059$ & $-0,054$ & $-0,009$ & -0.118 & 0.054 & -0.007 \\
\hline $\begin{array}{c}\text { Pointing Deviation } \\
\text { (degrees) }\end{array}$ & 0,066 & 0,046 & 0.048 & 0.066 & 0.066 & 0.056 \\
\hline
\end{tabular}

\subsection{Second approach: without daily updated data}

For the SCD1 satellite the simulation was performed considering all torques actuating together. The results, in terms of the difference between computed and actual right ascension, declination and spin velocity and pointing deviation, are shown in Tab. 5. The results show a good agreement between the computed values and the actual satellite behavior only for 1 day simulation. For more than 1 day the mean deviation error for the right ascension is higher than the precision required for INPE's SCC $\left(0.5^{\circ}\right)$. 
Table 5. Deviation between computed values and actual values when all torques actuating together for SCD1, without daily updated data

\begin{tabular}{|c|r|r|r|c|}
\hline Day & Right ascension & \multicolumn{1}{|c|}{ Declination } & \multicolumn{1}{c|}{ Spin velocity } & Pointing deviation \\
\hline $\mathbf{1 7 / 0 8 / 9 3}$ & 0 & 0 & 0 & 0 \\
\hline $\mathbf{1 8 / 0 8 / 9 3}$ & -0.871 & 0.346 & 0.021 & 0.373 \\
\hline $\mathbf{1 9 / 0 8 / 9 3}$ & -1.511 & 0.702 & 0.035 & 0.741 \\
\hline Mean & -0.794 & 0.349 & 0.0219 & 0.371 \\
\hline
\end{tabular}

Table 6 presents the results obtained when the same simulation is applied to SCD2. The results show a good agreement between the computed values and the actual satellite behavior for the entire simulated time interval of 11 day.

Table 6. Deviation between computed values and actual values when all torques actuating together for SCD2, without daily updated data

\begin{tabular}{|c|r|r|r|c|}
\hline Day & $\begin{array}{c}\text { Right ascension } \\
\text { (degrees) }\end{array}$ & \multicolumn{1}{c|}{$\begin{array}{c}\text { Declination } \\
\text { (degrees) }\end{array}$} & $\begin{array}{c}\text { Spin velocity } \\
\text { (rpm) }\end{array}$ & $\begin{array}{c}\text { Pointing deviation } \\
\text { (degrees) }\end{array}$ \\
\hline $\mathbf{1 2 / 2 / 2 0 0 2}$ & 0 & 0 & 0 & 0 \\
\hline $\mathbf{1 3 / 2 / 2 0 0 2}$ & -0.023 & 0.018 & 0.012 & 0.021 \\
\hline $\mathbf{1 4 / 2 / 2 0 0 2}$ & -0.048 & 0.029 & 0.014 & 0.036 \\
\hline $\mathbf{1 5 / 2 / 2 0 0 2}$ & -0.077 & 0.026 & 0.026 & 0.043 \\
\hline $\mathbf{1 6 / 2 / 2 0 0 2}$ & -0.104 & 0.008 & 0.028 & 0.047 \\
\hline $\mathbf{1 7 / 2 / 2 0 0 2}$ & -0.143 & -0.027 & 0.227 & 0.069 \\
\hline $\mathbf{1 8} / \mathbf{2} / \mathbf{2 0 0 2}$ & -0.196 & -0.084 & 0.241 & 0.122 \\
\hline $\mathbf{1 9} / \mathbf{2} / \mathbf{2 0 0 2}$ & & -0.116 & & 0.146 \\
& -0.198 & -0.16 & 0.253 & \\
\hline $\mathbf{2 0 / 2 / 2 0 0 2}$ & -0.211 & -0.216 & 0.265 & 0.186 \\
\hline $\mathbf{2 1 / 2 / 2 0 0 2}$ & -0.238 & -0.257 & 0.275 & 0.241 \\
\hline $\mathbf{2 2 / 2 / 2 0 0 2}$ & -0.212 & -0.311 & 0.288 & 0.274 \\
\hline $\mathbf{2 3 / 2 / 2 0 0 2}$ & -0.196 & -0.091 & 0.309 & 0.323 \\
\hline Mean & -0.137 & & 0.110 & 0.126 \\
\hline
\end{tabular}

\section{Conclusions}

In this paper a numerical approach was presented to the spin-stabilized satellite attitude propagation taking into account the residual torque, eddy current torque, aerodynamic torque, torque due to the Lorentz force and gravity gradient torque. The modeling scheme is entirely developed in terms of quaternion parametrization.

The theory was applied to the spin stabilized Brazilian's satellites SCD1 and SCD2. The results show that, for SCD1 and SCD2, the influence of the eddy current torque is larger than the others, not only due to the orbit altitude, but also due to the other specific satellites characteristics. The influence of the torque due to the Lorentz force is smaller than the other ones because of the dimension and the electrical charges of the SCD1 and SCD2.

Two approaches were presented. In the first one, where the attitude and orbital data are daily updated with real attitude data supplied by INPE, the results show a good agreement between the computed and actual data during the simulated time interval. The mean pointing deviation was $0.303^{\circ}$ for the SCD1 and of $0.056^{\circ}$ for the SCD2, which are within the dispersion range of the attitude determination system used for these satellites.

In the second approach, the attitude and orbital data are not daily updated. For SCD1, the results obtained showed a good agreement between the analytical solution and the actual satellite behavior only for one day simulation. For more than 1 day the mean deviation of the right ascension, declination and pointing deviation were higher than the accuracy required for SCC $\left(0.5^{\circ}\right)$. For the satellite SCD2, over the test period of 11 days, the difference between computed values and actual data remained within an acceptable dispersion range over all simulated time interval.

\section{Acknowledgments}

This present work was supported by CNPq (National Counsel of Technological and Scientific Development). 


\section{References}

Abdel-Aziz, Y. A., “Attitude Stabilization of a Rigid Spacecraft in the Geomagnetic Field”, Advances in Space Research, Elsevier Science Ltda, Vol. 40, pp. 30-36, 2007.

Beletskii, V.V. and Khentov, A. A., "Rotational Motion of a Magnetized Satellite”, Moscou, 1985.

Bento, P. R. S. P.,” Propagação da Atitude de Satélites Artificiais com Quatérnions e Torque Devido à Força de Lorentz”, Relatório Final de Bolsa de Iniciação Científica do CNPq/INPE, INPE library on-line INPE, São José dos Campos, 2010.

Garcia, R. V., Zanardi, M. C. and Kuga, H. K., "Spin-Stabilized Spacecrafts: Analytical Attitude Propagation using Magnetic Torques”, Math. Prob. Eng., VOL. 2009, pp. 1-19, 2009.

Lafontaine, J., and Mamem, R., “Orbit Lifetime Prediction and Safety Considerations”, IAF paper 84-269, 1984.

Pisacane, V. L., and Moore, R. C., “ Fundamentals of Space System”, Oxford University Press, New York, 1994.

Sehnal, L., and Pospísilová, L., “Thermospheric Model TD 88”, Publications from Astronomical Institute of the Czechoslovak Academy of Sciences, Observatory Ondrejov, Czechoslovakia, 1-9,1988.

Thomas, L. C. and Cappelari, J. O., "Attitude Determination and Prediction of Spin-stabilized Satellites", The Bell System Technical Journal, July 1964.

Vilhena de Moraes, R., “Non-Gravitational Disturbing Forces”, Adv. Space Res., VOL. 14, No. 5, pp. 45$68,1994$.

Wertz, J. R., “Spacecraft Attitude Determination and Control”, D. Reidel, Dordrecht, Holanda, 1978.

Zanardi, M. C., Quirelli, I. M. P. and Kuga, H. K., "Analytical Attitude Prediction of Spin Stabilized Spacecrafts Perturbed by Magnetic Residual Torques”, Adv. Spa. Res., VOL. 36, pp. 460-465, 2005.

Zanardi, M. C. and Real, F. F., "Environmental Torques Actuating on a Low Earth Orbiter Cylindrical Spacecraft”, Adv. in Sp. Res., Vol. 31, No. 8, pp. 1981-1986, Elsevier Ltd., 2003.

Zanardi, M. C., Silva, M. F. and Orlando, V., "Parametrização e Predição da Atitude de Satélites com Quaternions”, Anais do $8^{\circ}$ Congresso Brasileiro de Dinâmica, Controle e Aplicações, VOL. 1, pp.1-7, 2009.

Zanardi, M. C., Bento, P. R. S. P. and Orlando, V., "Predição da Orientação Espacial de Satélites Artificiais com Quaternions e Torques Ambientais”, Anais do CONEM 2010 v. 1, 1-10, CD_ROW, 2010. 